\title{
Relación longitud-peso del Perico (Trachelyopterus badeli Dahl, 1955) en el embalse de Urrá, Colombia
}

\section{Length-weight relationship of Perico (Trachelyopterus badeli Dahl, 1955) in Urra's dam, Colombia}

\author{
Charles W. Olaya-Nieto*, Fredys F. Segura-Guevara*, Indira M. de Hoyos-Negrete* \\ Resumen
}

\begin{abstract}
Se estimó la relación longitud-peso del Perico (Trachelyopterus badeli Dahl, 1955) en el embalse de Urrá, Alto río Sinú, Colombia, entre enero y diciembre del año 2003. La relación longitud-peso y el factor de condición se estimaron con $\mathrm{WT}=\mathrm{aL}^{\mathrm{b}}$ y $\mathrm{FC}=\mathrm{WT} / \mathrm{L}^{\mathrm{b}}$. La relación longitud total-peso total anual estimada fue WT=0.005 $( \pm 0.11) \mathrm{LT}^{3.31}{ }^{( \pm 0.09)}, \mathrm{r}=0.95, \mathrm{n}=608, \mathrm{y}$ su coeficiente de crecimiento fue estadísticamente diferente de 3.0. La relación longitud estándar-peso total anual estimada fue WT=0.015 $( \pm 0.12) \mathrm{LS}^{3.09}( \pm 0.10), r=0.93, \mathrm{n}=608$, y su coeficiente de crecimiento fue estadísticamente similar a 3 . Se encontraron diferencias estadísticas significativas entre los coeficientes de crecimiento mensuales tanto para LT-WT como LS-WT y se encontró correlación entre el factor de condición y el ciclo hidrológico del embalse de Urrá, en el año de estudio.
\end{abstract}

Palabras clave: Crecimiento; Factor de condición; Peces omnívoros; Hidroeléctrica; Río Sinú.

\begin{abstract}
The length-weight relationship of Perico (Trachelyopterus badeli Dahl, 1955) in the Urra's dam, Upper Sinu River, Colombia, between January and December 2003 was estimate. The length-weight relationship $\mathrm{TW}=\mathrm{aL}{ }^{\mathrm{b}}$ and condition factor $\mathrm{C} f=\mathrm{TW} / \mathrm{L}^{\mathrm{b}}$ were estimated. Total length-weight relationship estimated was $\mathrm{TW}=0.005( \pm 0.11) \mathrm{TL}^{3.31( \pm 0.09)}, \mathrm{r}=0.95, \mathrm{n}=608$, and its growth coefficient was statistically different from 3 . Standard length-weight relationship estimated was TW $=0.015( \pm 0.12) S L^{3.09( \pm 0.10)}, r=0.93, n=608$, and its growth coefficient was statistically similar to 3 . Statistically significant differences between monthly growth coefficients were found and correlation between the condition factor and the hydrological cycle of Urra's dam in the study year was observed.
\end{abstract}

Keywords: Growth; Condition factor; Omnivorous fishes; Hydroelectric; Sinu River.

\section{Introducción}

A finales del año 1999 se inició el llenado del embalse de Urrá y en febrero del año 2000 la Hidroeléctrica Urrá comenzó la generación de energía eléctrica, creándose un nuevo ecosistema en el río Sinú: el embalse. Estas nuevas condiciones implicaron, alteraciones en la calidad del agua vertida en el tramo adyacente aguas abajo y la regulación de los caudales del río que podrían afectar la dinámica poblacional de los peces reofílicos (Olaya-Nieto et al. 2000).
El embalse ha sido considerado como un cuerpo de agua con amplias posibilidades para su aprovechamiento pesquero, lo cual redundaría en beneficio de las comunidades reasentadas que habitan en su área de influencia. El proyecto monitoreo ictiológico y pesquero del embalse de Urrá registró 42 especies entre reofílicas y residentes o sedentarias (Valderrama et al. 2002), dentro de las cuales se encuentra el Perico (Trachelyopterus badeli Dahl, 1955), un pez de cuero de pequeño tamaño, con hembras más grandes que los machos, alcanzando tallas hasta de $23 \mathrm{~cm}$ de longitud total, poca importancia

* Laboratorio de Investigación Biológico Pesquera (LIBP), Universidad de Córdoba, Departamento de Ciencias Acuícolas, Lorica, Colombia. e-mail: charles_olaya@hotmail.com

Fecha recepción: Febrero 12, $2012 \quad$ Fecha aprobación: Mayo 10, 2012 
comercial en la cuenca del río Sinú, pero con posibilidades en la acuariofilia porque se adapta al cautiverio en tanques de cemento o acuarios de vidrio mostrando conducta gregaria no agresiva (OlayaNieto et al. 2004).

$\mathrm{Al}$ igual que otros peces nativos de la cuenca ha sido poco estudiado, por lo que en la actualidad son escasos los reportes sobre su crecimiento en talla y peso (De Hoyos 2008), aspecto muy importante teniendo en cuenta que el manejo y desarrollo del recurso pesquero en los embalses se hace dentro de un enfoque integrado que involucre el aspecto pesquero, armonizándolo con los criterios ambientales y que responda a los intereses de los usuarios a fin de generar acciones que permitan garantizar el principio de equidad (FAO 1998).

El concepto de pesca responsable plantea una serie de orientaciones técnicas que incluyen a los embalses como sistemas de manejo relevantes (FAO 1998), por lo que se deben realizar estudios que con- duzcan al ordenamiento de la pesquería y del embalse de Urrá en sí para que las poblaciones humanas asentadas allí puedan realizar faenas de pesca de subsistencia o comercial, cuyo consumo o comercialización contribuya con su seguridad alimentaria.

El objetivo de esta investigación fue establecer la relación longitud-peso del Perico en el embalse de Urrá, río Sinú, como contribución a la comprensión de su ciclo de vida y al ordenamiento de su pesquería.

\section{Metodología}

Descripción del área de estudio. El estudio se desarrolló en el embalse de Urrá (Figura 1), localizado al sur del departamento de Córdoba, al noroccidente de Colombia y a $30 \mathrm{~km}$ del municipio de Tierralta. Este embalse represó al río Sinú a la altura de Angostura de Urrá a 137 msnm a 297 km de su desembocadura con un área de 7400 Ha y for-



Figura 1. Localización geográfica del embalse de Urrá. 
ma dendriforme por la geomorfología de las colas embalsadas de los ríos y quebradas que le aportan sus aguas (Valderrama et al. 2006). Sus principales afluentes son los ríos Verde, Esmeralda, Manso y Tigre, que nacen en el Parque Nacional Natural Paramillo y confluyen aguas arriba del embalse, constituyendo el principal aporte hidrológico al mismo en conjunto con algunas quebradas como las de Tucurá, Urrá y Naín (Valderrama et al. 2006). El Parque Nacional Natural Paramillo tiene jurisdicción sobre un área del embalse (cola del río Verde) y el embalse como tal hace parte de la zona de amortiguación del parque.

Para la identificación taxonómica de la especie se aplicaron las claves propuestas por Dahl (1971). Utilizando línea de mano y trasmallo se capturaron individuos entre enero y diciembre 2003. A cada individuo se le tomó longitud total (LT) y longitud estándar (LS) al milímetro más cercano con un ictiómetro graduado y el peso total (WT) al gramo más cercano con una balanza eléctrica Ohaus con capacidad de $1500 \pm 0.01 \mathrm{~g}$ y se establecieron las distribuciones de frecuencias de tallas y pesos para el total de muestras.

Relación longitud-peso. La relación longitudpeso es una regresión potencial que relaciona una medida lineal (talla) con una de volumen (peso) de acuerdo con la ecuación

$\mathrm{WT}=\mathrm{aL}^{\mathrm{b}}$ (Ricker 1975), donde WT es el peso total del pez en gramos, a es una constante de regresión equivalente al factor de condición $(\mathrm{Fc}), \mathrm{L}$ es la longitud total (LT) o estándar (LS) en centímetros y b es el coeficiente de crecimiento de la regresión.

Factor de condición. El Fc se estimó con la ecuación $\mathrm{Fc}=\mathrm{WT} / \mathrm{L}^{\mathrm{b}}$ (Bagenal y Tesch 1978). El procedimiento fue el siguiente: se estimó la relación longitud-peso para un mes determinado, de donde se obtuvieron los valores alcanzados por el coeficiente de crecimiento (b) y el Fc. Para estimar el Fc diario o de una muestra cualquiera, se utilizó la talla y peso de esta muestra y se reemplazó el coeficiente de crecimiento estimado para el mes al que pertenece la muestra en la ecuación que aparece arriba.

Los valores obtenidos se expresaron como promedio ( \pm desviación estándar), se establecieron intervalos de confianza al 95\%, se estimaron los coeficientes de correlación ( $\mathrm{r}$ ) y de determinación $\left(\mathrm{r}^{2}\right)$ para la relación lineal y para la relación longitud- peso, y se aplicó el test de Student al coeficiente de crecimiento (b) de la relación longitud-peso para establecer si era isométrico o no. También se aplicó la técnica del análisis de varianza de una vía a las pendientes de las relaciones lineales y a los coeficientes de crecimiento de las relaciones longitudpeso, y la prueba de comparaciones múltiples de Tukey-Kramer cuando se encontraron diferencias estadísticas significativas entre ellas.

\section{Resultados}

Se colectaron 608 individuos, 284 hembras, 320 machos y cuatro indiferenciados, por lo que la proporción sexual total hembra: macho fue de 1.0:1.1, similar a lo esperado $\left(\mathrm{X}^{2}: 2.146 ; \mathrm{p}: 0.05 ; 1 \mathrm{gl}\right)$. La longitud estándar de los individuos osciló entre 6.6$22.8(16.8 \pm 2.1) \mathrm{cm}$, con intervalo de confianza de $95 \%$ de 0.17 y coeficiente de variación de $12.5 \%$; la longitud total osciló entre 8.1-26.5 $(19.5 \pm 2.3) \mathrm{cm}$, con intervalo de confianza de 0.2 y coeficiente de variación de $12 \%$. La distribución de frecuencias de tallas mostró rangos entre 8 y $27 \mathrm{~cm}$ LT (Figura 2), con talla media de captura de $19.5 \mathrm{~cm}$ LT. Como todos los coeficientes de variación son menores del $30 \%$, se infiere que las tallas muestreadas son homogéneas.

El peso fluctuó entre 5 y $288(99.4 \pm 44.2) \mathrm{g}$, con intervalo de confianza de $95 \%$ de 3.5 . El coeficiente de variación fue mayor del $30 \%$ en todos los casos, mostrando valores de $31 \%$ (noviembre) hasta $63.8 \%$ (enero), con promedio anual de $44.5 \%$, lo que indica que hay heterogeneidad entre los pesos. La distribución de frecuencias de pesos mostró rangos entre 25 y 275 g (Figura 3 ), con peso medio captura de 101.6 g.

Los parámetros de crecimiento de la relación longitud-peso se estimaron a partir de la información mensual de longitud total, longitud estándar y peso total (Tablas 1 y 2), mientras que en la Tabla 3 se presenta la información anual para hembras, machos y sexos combinados.

En la relación longitud total-peso total, el coeficiente de crecimiento b fluctuó entre $2.92( \pm 0.30)$ en el mes de diciembre y $3.81( \pm 0.55)$ en el mes de enero (Tabla 1), con valor anual de $3.31( \pm 0.09)$. El test de Student $(\mathrm{p}<0.05)$ confirmó que seis coeficientes de crecimiento mensual son estadísticamente si- 


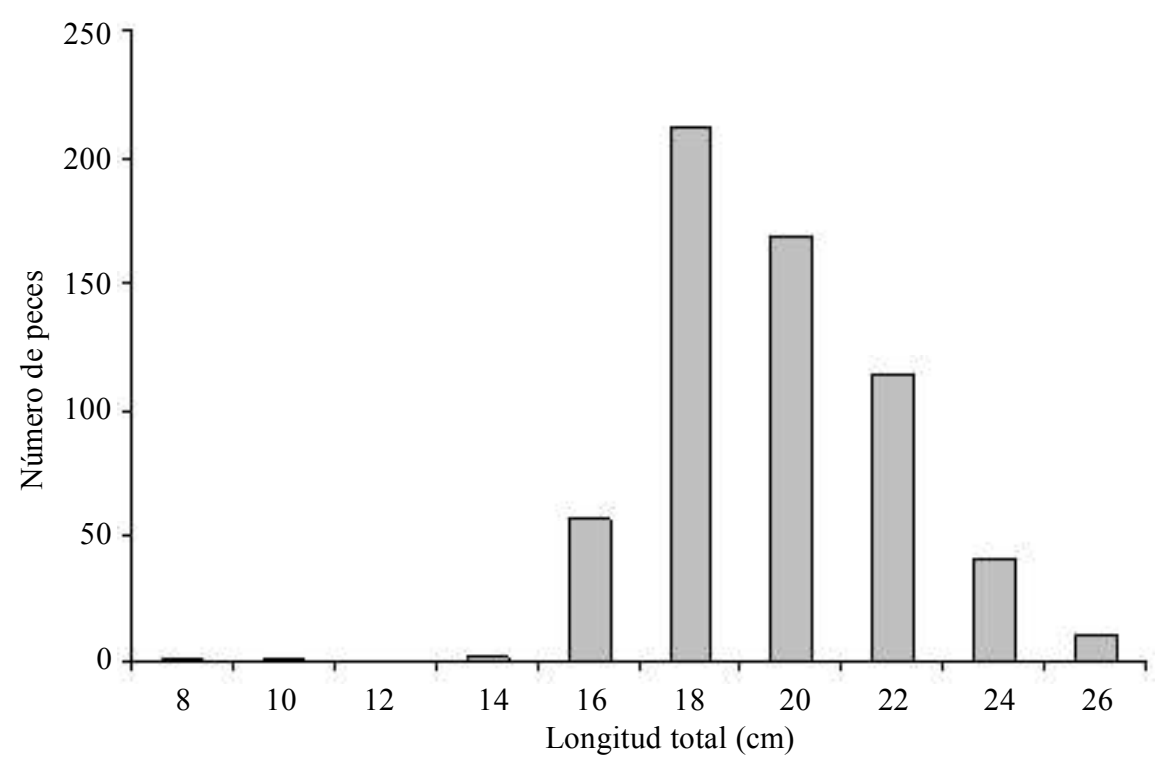

Figura 2. Distribución de frecuencia de tallas del Perico en el embalse de Urrá.

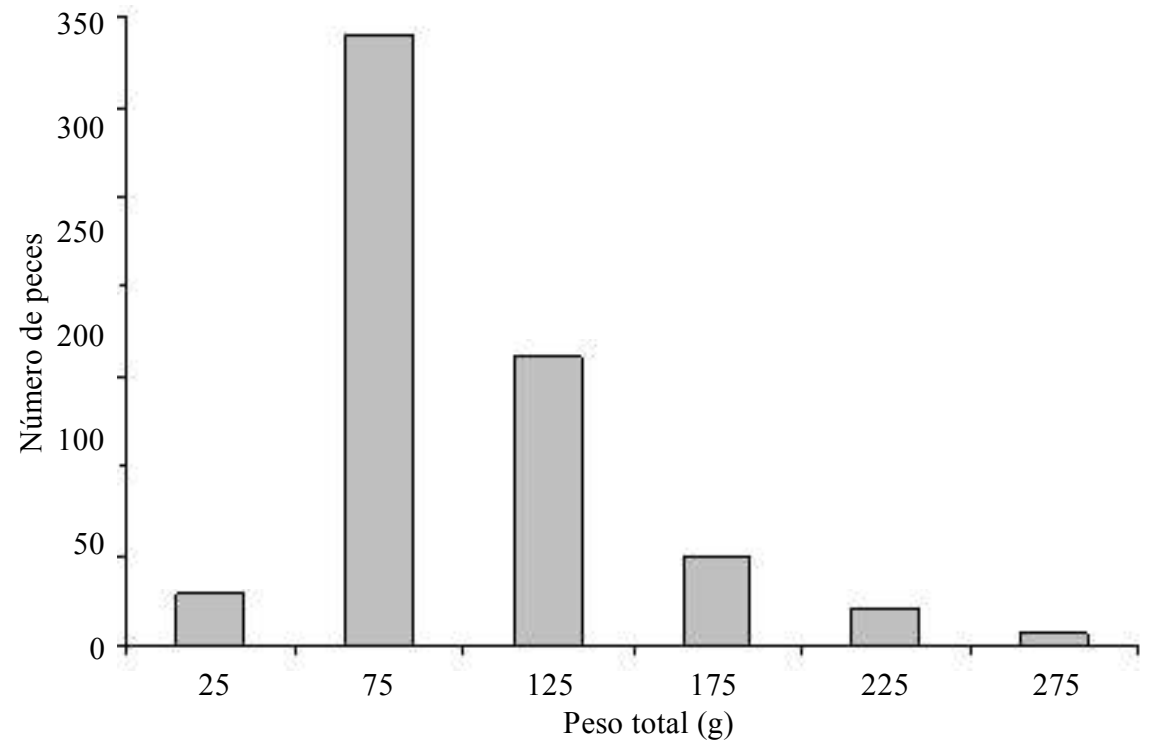

Figura 3. Distribución de frecuencia de pesos del Perico en el embalse de Urrá.

milares a tres o isométricos (febrero, marzo, abril, agosto, noviembre y diciembre) y el resto son mayores y estadísticamente diferentes de tres o alométricos positivos (enero, mayo, junio, julio, septiembre, octubre). El coeficiente de crecimiento anual también fue estadísticamente diferente de 3 , es decir, alométrico positivo.

Para la relación longitud estándar-peso total, el coeficiente de crecimiento b fluctuó entre 2.61 $( \pm 0.40)$ en el mes de agosto y $3.88( \pm 0.78)$ en el mes de enero (Tabla 2), con valor anual $3.09( \pm 0.10)$. El test de Student $(\mathrm{p}<0.05)$ confirmó que la mayoría de los coeficientes de crecimiento mensual son isométricos, excepto enero y mayo que son alométricos positivos, mientras que el coeficiente de crecimiento anual fue isométrico

Aplicando el análisis de varianza se encontraron diferencias estadísticas significativas entre los coeficientes de crecimiento mensual tanto para la longitud total $(\mathrm{F}=86.004 ; \mathrm{p}=0.0001 ; \mathrm{gl}=607)$ como la 
Tabla 1. Información básica de talla, peso y parámetros de crecimiento de la relación longitud-peso (LT-WT) sexos combinados del Perico, año 2003.

\begin{tabular}{|c|c|c|c|c|c|c|c|c|c|c|c|}
\hline \multirow[t]{2}{*}{ Meses } & \multicolumn{4}{|c|}{ Longitud total (cm) } & \multicolumn{3}{|c|}{ Peso total (g) } & \multicolumn{4}{|c|}{ Relación longitud-peso } \\
\hline & $\mathbf{n}$ & rango & Prom & $\mathrm{DE}$ & rango & Prom & DE & $b( \pm I C)$ & $a( \pm I C)$ & $\mathbf{r}$ & $\mathbf{r}^{2}$ \\
\hline Enero & 10 & $14.2-23.5$ & 19.4 & \pm & 25.0 & 1 & \pm 73.9 & $.55)$ & 0.001( & 0.98 & 0.97 \\
\hline Febrero & 10 & $15.2-23.0$ & 17.9 & \pm 2.5 & $39.0-180.0$ & 77.5 & \pm 43.8 & $3.50( \pm 0.75)$ & $0.003( \pm 0.93)$ & 0.97 & 0.94 \\
\hline Marzo & 29 & $15.0-24.6$ & 17.9 & \pm 2.8 & $39.0-259.0$ & 81.1 & \pm 51.2 & $3.25( \pm 0.28)$ & $0.006( \pm 0.35)$ & 0.98 & 0.95 \\
\hline Abril & 34 & $15.4-26.5$ & 20.5 & \pm 3.0 & $37.0-288.0$ & 131.1 & \pm 65.1 & $3.29( \pm 0.33)$ & $0.006( \pm 0.44)$ & 0.96 & 0.93 \\
\hline Mayo & 44 & $16.1-25.2$ & 20.2 & \pm 2.6 & $49.0-257.0$ & 115.1 & \pm 59.1 & $3.66( \pm 0.31)$ & $0.002( \pm 0.40)$ & 0.96 & 0.93 \\
\hline Junio & 38 & $14.3-25.5$ & 19.5 & \pm 2.9 & $37.0-279.0$ & 102.1 & \pm 58.4 & $3.35( \pm 0.22)$ & $0.004( \pm 0.28)$ & 0.98 & 0.97 \\
\hline Julio & 28 & $15.4-24.0$ & 19.2 & \pm 2.8 & $34.0-212.0$ & 100.9 & \pm 54.1 & $3.54( \pm 0.33)$ & $0.003( \pm 0.43)$ & 0.97 & 0.95 \\
\hline Agosto & 86 & $15.3-23.2$ & 19.0 & \pm 1.8 & $45.0-193.0$ & 93.5 & \pm 31.2 & $3.15( \pm 0.35)$ & $0.008( \pm 0.44)$ & 0.89 & 0.79 \\
\hline Septiembre & 103 & $10.0-26.2$ & 19.5 & \pm 2.1 & $10.0-258.0$ & 94.9 & \pm 37.7 & $3.27( \pm 0.23)$ & $0.006( \pm 0.30)$ & 0.94 & 0.89 \\
\hline Octubre & 115 & $8.1-25.3$ & 19.3 & \pm 2.0 & $5.0-202.0$ & 90.3 & \pm 32.1 & $3.32( \pm 0.21)$ & $0.005( \pm 0.27)$ & 0.95 & 0.90 \\
\hline Noviembre & 50 & $16.3-23.4$ & 20.1 & \pm 1.9 & $47.0-170.0$ & 100.7 & \pm 31.2 & $3.28( \pm 0.38)$ & $0.005( \pm 0.50)$ & 0.93 & 0.88 \\
\hline Diciembre & 61 & $17.1-25.4$ & 20.3 & \pm 1.9 & $52.0-273.0$ & 109.5 & \pm 35.1 & $2.92( \pm 0.30)$ & $0.016( \pm 0.40)$ & 0.93 & 0.86 \\
\hline
\end{tabular}

Tabla 2. Información básica de talla, peso y parámetros de crecimiento de la relación longitud-peso (LS-WT) sexos combinados del Perico, año 2003.

\begin{tabular}{|c|c|c|c|c|c|c|c|c|c|c|c|}
\hline \multirow[t]{2}{*}{ Meses } & \multicolumn{4}{|c|}{ Longitud total (cm) } & \multicolumn{3}{|c|}{ Peso total (g) } & \multicolumn{4}{|c|}{ Relación longitud-peso } \\
\hline & $\mathbf{n}$ & rango & Prom & DE & rango & Prom & DE & $b( \pm I C)$ & $a( \pm I C)$ & $\mathbf{r}$ & $\mathbf{r}^{2}$ \\
\hline Enero & 10 & $11.9-20.5$ & 16.5 & \pm 2.9 & $30.0-225.0$ & 115.9 & \pm 73.9 & $3.88( \pm 0.78)$ & $0.002( \pm 0.94)$ & 0.97 & 0.94 \\
\hline Febrero & 10 & 12.6-19.4 & 14.9 & \pm 2.3 & $39.0-180.0$ & 77.5 & \pm 43.8 & $3.28( \pm 0.64)$ & $0.010( \pm 0.76)$ & 0.97 & 0.95 \\
\hline Marzo & 29 & $12.8-21.5$ & 15.3 & \pm 2.4 & $39.0-259.0$ & 81.1 & \pm 51.2 & $3.24( \pm 0.34)$ & $0.011( \pm 0.40)$ & 0.97 & 0.94 \\
\hline Abril & 34 & $13.0-22.0$ & 17.3 & \pm 2.6 & $37.0-288.0$ & 131.1 & \pm 65.1 & $3.19( \pm 0.30)$ & $0.014( \pm 0.37)$ & 0.97 & 0.93 \\
\hline Mayo & 44 & $13.7-21.7$ & 17.2 & \pm 2.3 & $49.0-257.0$ & 115.1 & \pm 59.1 & $3.59( \pm 0.29)$ & $0.004( \pm 0.36)$ & 0.97 & 0.93 \\
\hline Junio & 38 & 11.8-22.6 & 16.8 & \pm 2.6 & $37.0-279.0$ & 102.1 & \pm 58.4 & $3.25( \pm 0.25)$ & $0.010( \pm 0.30)$ & 0.98 & 0.95 \\
\hline Julio & 28 & $12.8-20.6$ & 16.2 & \pm 2.4 & $34.0-212.0$ & 100.9 & \pm 54.1 & $3.41( \pm 0.50)$ & $0.007( \pm 0.60)$ & 0.94 & 0.88 \\
\hline Agosto & 86 & $12.8-20.5$ & 16.3 & \pm 1.7 & $45.0-193.0$ & 93.5 & \pm 31.2 & $2.61( \pm 0.40)$ & $0.061( \pm 0.48)$ & 0.82 & 0.67 \\
\hline Septiembre & 103 & $7.9-22.8$ & 16.9 & \pm 1.9 & $10.0-258.0$ & 94.9 & \pm 37.7 & $3.08( \pm 0.21)$ & $0.015( \pm 0.26)$ & 0.95 & 0.89 \\
\hline Octubre & 115 & $6.6-21.7$ & 16.8 & \pm 1.8 & $5.0-202.0$ & 90.3 & \pm 32.1 & $3.16( \pm 0.20)$ & $0.012( \pm 0.24)$ & 0.95 & 0.90 \\
\hline Noviembre & 50 & $14.1-20.6$ & 17.5 & \pm 1.7 & $47.0-170.0$ & 100.7 & \pm 31.2 & $3.11( \pm 0.33)$ & $0.013( \pm 0.41)$ & 0.94 & 0.88 \\
\hline Diciembre & 61 & $14.7-22.3$ & 17.7 & \pm 1.8 & $52.0-273.0$ & 109.5 & \pm 35.1 & $2.66( \pm 0.28)$ & $0.050( \pm 0.35)$ & 0.93 & 0.86 \\
\hline
\end{tabular}

Tabla 3. Información básica anual de talla, peso y parámetros de crecimiento de la relación longitud-peso del Perico, año 2003.

\begin{tabular}{|c|c|c|c|c|c|c|c|c|c|c|c|}
\hline \multirow[t]{2}{*}{ Sexo } & \multicolumn{4}{|c|}{ Longitud total $(\mathrm{cm})$} & \multicolumn{3}{|c|}{ Peso total (g) } & \multicolumn{4}{|c|}{ Relación longitud-peso } \\
\hline & $\mathbf{n}$ & rango & Prom & DE & rango & Prom & DE & $b( \pm I C)$ & $a( \pm I C)$ & $r$ & $\mathbf{r}^{2}$ \\
\hline Hembras & 284 & $15.0-26.5$ & 21.2 & \pm 2.0 & $37.0-288.0$ & 131.2 & \pm 44.4 & $3.17( \pm 0.15)$ & $0.008( \pm 0.20)$ & 0.93 & 0.86 \\
\hline Machos & 320 & $14.3-21.1$ & 18.1 & \pm 1.2 & $34.0-122.0$ & 72.0 & \pm 15.7 & $2.63( \pm 0.20)$ & $0.034( \pm 0.26)$ & 0.82 & 0.67 \\
\hline SC & 608 & $8.1-26.5$ & 19.5 & \pm 2.3 & $5.0-288.0$ & 99.4 & \pm 44.2 & $3.31( \pm 0.09)$ & $0.005( \pm 0.11)$ & 0.95 & 0.90 \\
\hline
\end{tabular}

estándar $(\mathrm{F}=202.45 ; \mathrm{p}=0.0001 ; \mathrm{gl}=607)$. Aplicando la prueba de Tukey-Kramer se observó que tales diferencias se presentaron entre todos los meses evaluados.

En la relación longitud total-peso total, el factor de condición osciló entre $0.001( \pm 0.70)$ en enero y $0.016( \pm 0.40)$ en diciembre (Tabla 1$)$, con valor anual de $0.005( \pm 0.11)$, sin diferencias estadísticas signifi- cativas entre ellos al aplicar el análisis de varianza $(\mathrm{F}=0.01953 ; \mathrm{p}=0.9999 ; \mathrm{gl}=607)$. En la relación longitud estándar-peso total, el factor de condición fluctuó entre $0.002( \pm 0.94)$ en enero y $0.061( \pm 0.48)$ en agosto (Tabla 2), con valor anual de $0.015( \pm 0.12)$, y tampoco se encontraron diferencias estadísticas significativas entre ellos al aplicar el análisis de varianza $(\mathrm{F}=0.5995 ; \mathrm{p}=0.8299 ; \mathrm{gl}=607)$. 
Con los valores obtenidos para el coeficiente de crecimiento (b) y el factor de condición ( $\mathrm{Fc}$ ) para el año en estudio se estimaron las relaciones longitud total-peso total WT $=0.005( \pm 0.11) \mathrm{LT}^{3.31}( \pm 0.09)$, $\mathrm{r}=0.95, \mathrm{n}=608$ (Figura 4) y longitud estándar-peso

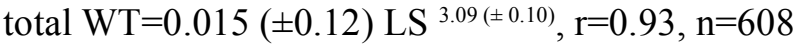
(Figura 5),donde el coeficiente de correlación es alto en ambos casos $(0.95,0.93)$ y estadísticamente significativo $(\mathrm{p}<0.05)$.

También se estimaron las relaciones longitud total-peso total para hembras WT $=0.008( \pm 0.20) \mathrm{LT}$ $3.17( \pm 0.15), \mathrm{n}=284, \mathrm{r}=0.93$ y machos $\mathrm{WT}=0.034( \pm 0.26)$

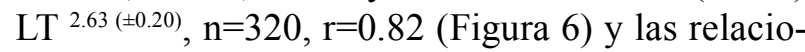
nes longitud estándar-peso total también para hembras y machos WT $=0.028( \pm 0.01) \operatorname{LS}^{2.89( \pm 0.18)}, \mathrm{n}=284$, $\mathrm{r}=0.88$ y WT$=0.124( \pm 0.22) \operatorname{LS}^{2.31}( \pm 0.19), \mathrm{n}=320$, $\mathrm{r}=0.81$ (Figura 7).

Con referencia al ciclo hidrológico del embalse de Urrá, en el año de estudio (2003) en varios meses se observó correlación entre el factor de condición

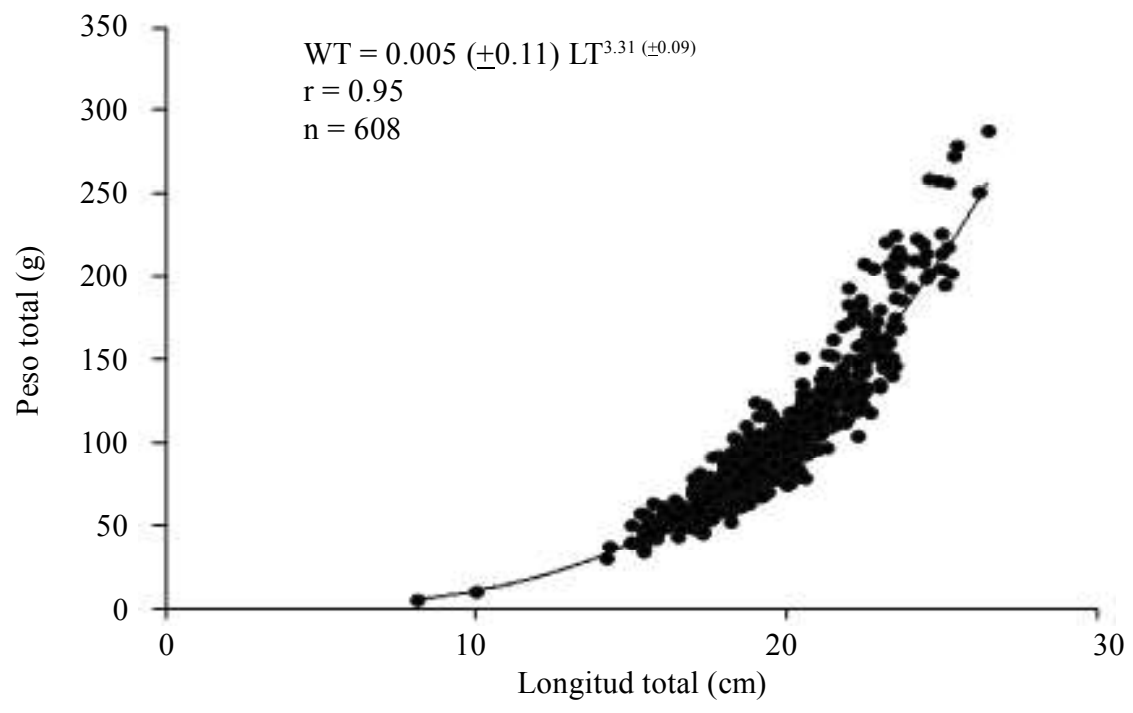

Figura 4. Relación longitud total-peso total del Perico en el embalse de Urrá. Sexos combinados.

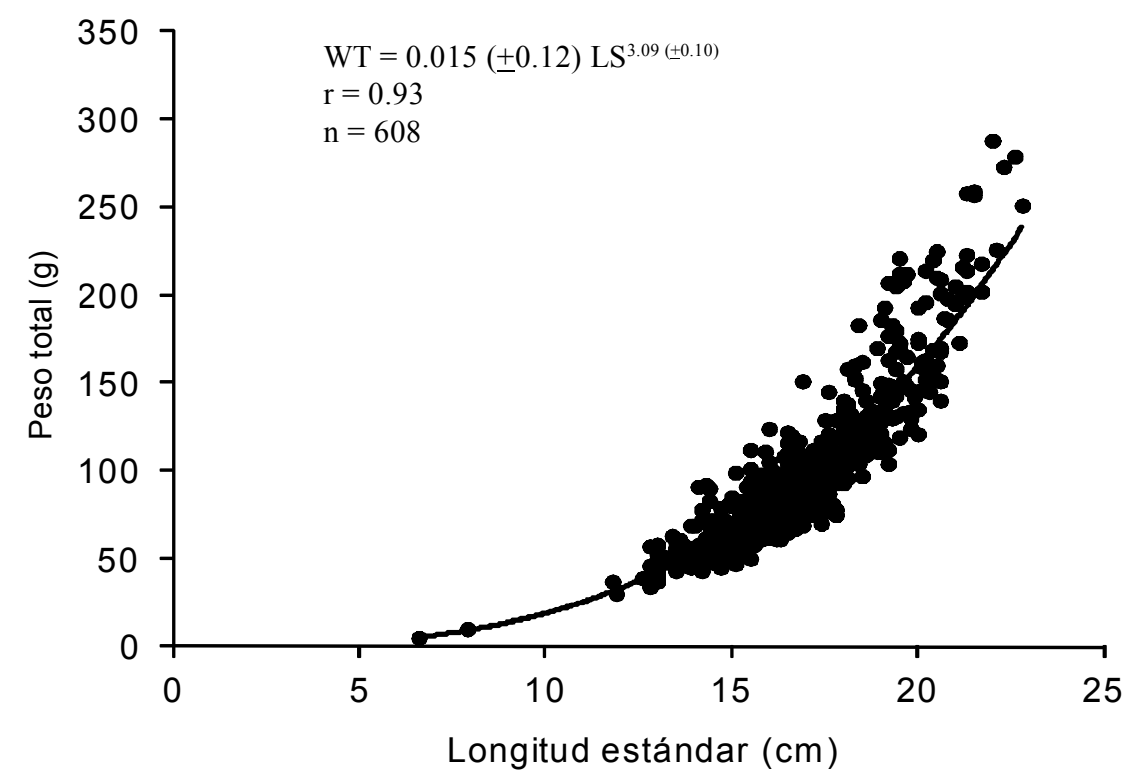

Figura 5. Relación longitud estándar-peso total del Perico en el embalse de Urrá. Sexos combinados. 

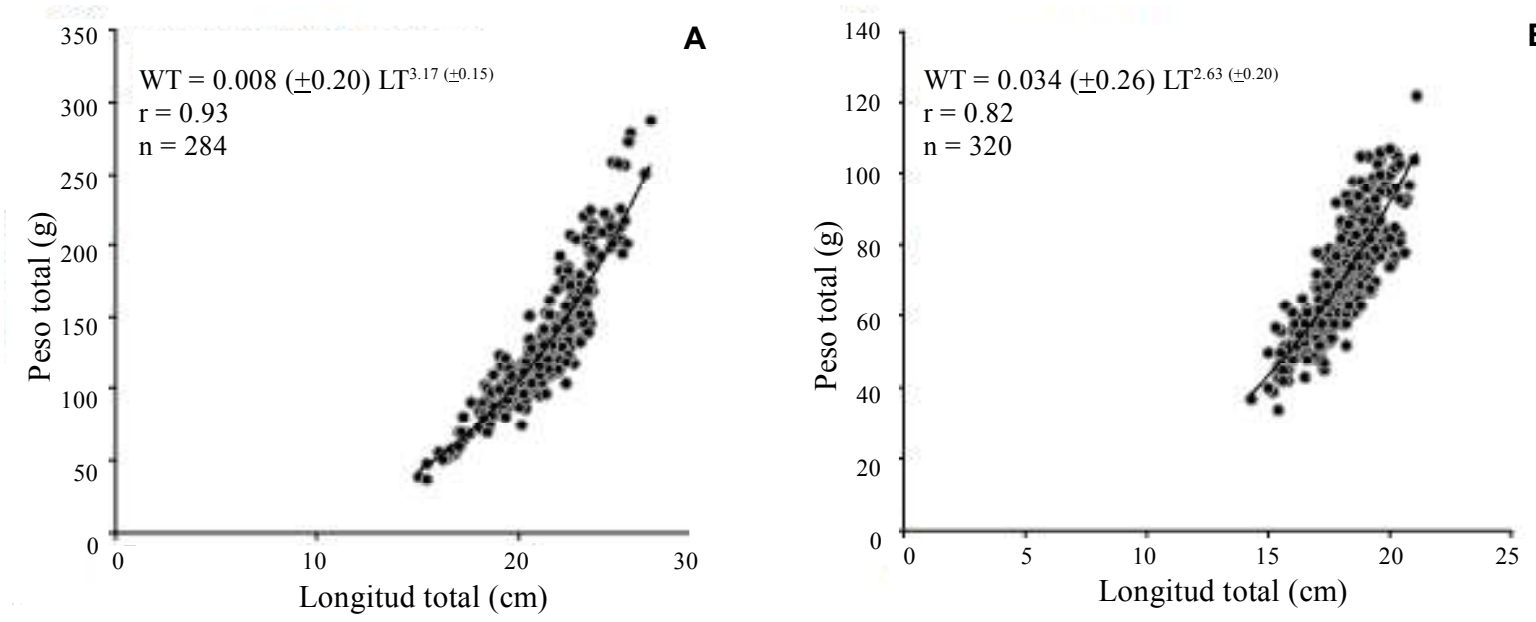

Figura 6. Relación longitud total-peso total del Perico en el embalse de Urrá. Hembras (A) y machos (B).
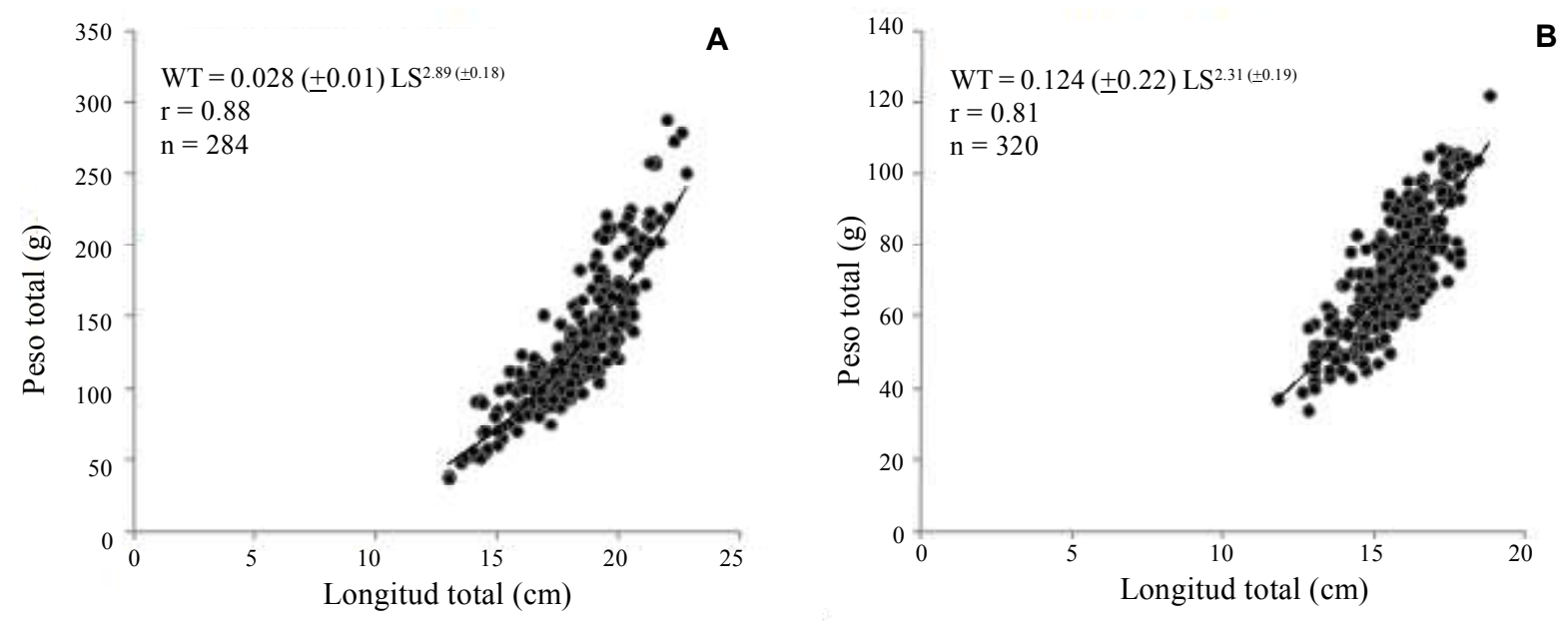

Figura 7. Relación longitud estándar-peso total del Perico en el embalse de Urrá. Hembras (A) y machos (B).

de la relación longitud-peso con los niveles del embalse.

\section{Discusión}

Se observó que la talla media de captura de Valderrama et al. (2003) (17.7 cm LS, $20.5 \mathrm{~cm} \mathrm{LT}$, $\mathrm{n}=479)$ es mayor que la estimada en este trabajo; la de Valderrama et al. (2004) (16.8 cm LS, $19.5 \mathrm{~cm}$ LT) es similar, mientras que la de Valderrama et al. (2006) (16.3 cm LS, $18.9 \mathrm{~cm} \mathrm{LT,} \mathrm{n}=316$ ) es menor, al igual que la de Ramírez y Saleme (2008) $(15.9 \mathrm{~cm}$ LT, $n=289$ ). Tales diferencias se pueden deber a la ubicación y distancia observada entre las áreas geográficas estudiadas, a la selectividad del arte de pesca utilizado por estos autores, al número de individuos colectados y las condiciones ambientales que presenta el embalse, el cual es un cuerpo de agua relativamente nuevo.

Como la talla es una magnitud lineal y el peso es proporcional a su cubo, durante el crecimiento de los peces la mayor parte de ellos tienen dimensiones diferentes; si el pez al crecer mantiene sus proporciones corporales y su gravedad especifica constante, se dice que el crecimiento es isométrico $(b=3)$, cuando b es mayor o menor que 3 , el crecimiento es alométrico y el pez llega a ser más pesado o menos pesado para la longitud que tiene (Bagenal y Tesch 1978).

Estudios realizados con diferentes especies y ambientes reportan que el valor del coeficiente de crecimiento en condiciones naturales oscila entre 2.5 y 4. Este coeficiente puede variar entre especies y 
entre poblaciones de la misma especie (Bagenal y Tesch 1978), y valores de b menores de 2.5 o mayores de 3.5 son estimados generalmente a partir de muy pequeños rangos de talla y/o se pueden considerar como estimaciones erradas (Pauly 1984). Olaya-Nieto et al. (2007) afirman que b es mayor que 3.5 o 4 cuando se trabaja con datos que corresponden a peces en avanzado estado de madurez sexual, sobre todo en hembras, como los meros y los pargos y los peces reofílicos cuando hacen agregaciones para desovar.

El coeficiente de crecimiento de la relación longitud-peso (LS-WT) estimado en este trabajo para sexos combinados (3.09) y para hembras (2.89) se encuentra dentro del rango 2.96-3.40, cuyo promedio \pm intervalo de confianza al $99 \%$ es $3.11( \pm 0.22)$, de acuerdo con los reportes de Benedito-Cecilio et al. (1997), Valderrama et al. (2003), Olaya-Nieto et al. (2004) y Pérez et al. (2007) para algunas especies de la familia Auchenipteridae, lo que no sucede con el valor estimado para machos (2.31), el cual no encaja dentro de este rango (Tabla 4).

Se confirmó la premisa de la relación inversa existente entre el coeficiente de crecimiento (b) y el Fc de la relación longitud-peso. El Fc es el grado de bienestar o condición somática de una especie en relación con el medio en que vive, conociéndose también como grado de robustez o índice ponderal. Es un buen indicador del crecimiento y un parámetro útil para conocer la conveniencia de un determinado ambiente sobre una especie (Gulland 1983), siendo más utilizado que el coeficiente de crecimiento (b), porque las variaciones observadas en este último son usualmente pequeñas, para indicar la condición o bienestar de los peces, basándose en la hipótesis de que los peces más pesados de una misma talla están en mejor condición que los menos pesados.

Durante el proceso de colonización de un reservorio o de un embalse, algunas poblaciones son reducidas, mientras otras muestran un crecimiento explosivo en respuesta a los rápidos cambios ambientales (Agostinho et al. 1997). Los cambios abruptos en la dinámica del agua conducen a modificaciones en las condiciones ambientales, alterando las interacciones bióticas y las proporciones de los recursos alimenticios (Hahn et al. 1997). De igual forma, el aporte alimenticio llega a ser un importante indicador del éxito de las especies durante la ocupación de un nuevo ambiente (Rodríguez-Ruiz 1998).

Aunque algunos peces en ambientes tropicales presentan marcadas especializaciones tróficas, la mayoría exhibe gran flexibilidad alimentaria (LoweMcConnell 1999). Esta plasticidad trófica puede ser una estrategia importante que conduzca al uso del abundante recurso alimenticio disponible al inicio de la formación, construcción o llenado de un reservorio o embalse (Luz-Agostinho et al. 2006). Sin embargo, el recurso alimenticio inicialmente abundante, sobre todo alóctono, tiende a disminuir después del primer año de llenado (Agostinho et al. 1999), promoviendo alteraciones en las poblaciones las cuales son reflejadas en la estructura de la comu-

Tabla 4. Parámetros de crecimiento de la relación longitud-peso del Perico y algunas especies de la familia Auchenipteridae en América del Sur.

\begin{tabular}{lcrrrrl}
\hline \multicolumn{1}{c}{ Especie } & $\begin{array}{c}\text { Medición } \\
\text { (cm) }\end{array}$ & $\mathbf{a}$ & $\mathbf{b}$ & $\mathbf{n}$ & $\mathbf{r}$ & \multicolumn{1}{c}{ Fuente } \\
\hline Ageneiosus brevifilis - SC & LS & 0,0200 & 2,98 & 32 & 0,99 & Benedito-Cecilio et al. 1997 \\
Ageneiosus caucanus - SC & LT & 0,0020 & 3,40 & 416 & 0,98 & Pérez et al., 2007 \\
Ageneiosus ucayalensis - SC & LS & 0,0107 & 2,97 & 2476 & 0,96 & Benedito-Cecilio et al. 1997 \\
Ageneiosus valenciennesi -SC & LS & 0,0067 & 3,19 & 88 & 0,98 & Benedito-Cecilio et al. 1997 \\
Auchenipterus nuchalis - SC & LS & 0,0150 & 2,96 & 128.867 & 0,94 & Benedito-Cecilio et al. 1997 \\
Parauchenipterus galeatus - SC & LS & 0,0337 & 2,99 & 3578 & 0,95 & Benedito-Cecilio et al. 1997 \\
Trachelyopterus insignis - SC & LS & $3 \times 10^{-6}$ & 3,35 & 479 & 0,96 & Valderrama et al. 2003 \\
Trachelyopterus badeli - SC & LS & 0,021 & 3,07 & 289 & 0,91 & Olaya-Nieto et al. 2004 \\
Trachelyopterus badeli - SC & LS & 0,015 & 3,09 & 608 & 0,93 & Este trabajo, 2008 \\
Trachelyopterus badeli - H & LS & 0,028 & 2,89 & 284 & 0,88 & Este trabajo, 2008 \\
Trachelyopterus badeli - M & LS & 0,124 & 2,31 & 320 & 0,81 & Este trabajo, 2008 \\
\hline
\end{tabular}

$\mathrm{SC}=$ sexos combinados, $\mathrm{H}=$ hembra, $\mathrm{M}=$ macho, ${ }^{*}=$ talla en $\mathrm{mm}$. 
nidad (Luz-Agostinho et al. 2006).

Por lo tanto, son fundamentales la descripción de la estructura trófica de las comunidades de peces y el conocimiento de las relaciones alimenticias entre los organismos para una efectiva conservación y manejo de la fauna íctica. La información de la dieta de las comunidades de peces a escalas espacial y temporal, cubriendo el desarrollo ontogénico de las especies, aporta información importante sobre la disponiblidad de los recursos (Winemiller, 1986) y sobre el uso de tales recursos (Agostinho et al. 1997).

A grandes rasgos, esto es lo que probablemente ha venido sucediendo en el embalse de Urrá desde su construcción y llenado, lo que ya ha sido observado por Valderrama et al. (2006) quienes reportaron la disminución de peces detritívoros, los cuales fueron sustituidos por peces omnívoros; como el Perico, una de las especies que mejor se ha adaptado a las condiciones físicas, químicas y biológicas del embalse, por su plasticidad trófica, lo que también explicaría el alto valor alcanzado por el factor de condición o índice de bienestar de la especie en estudio.

\section{Agradecimientos}

Los autores expresan sus agradecimientos a los pescadores y comercializadores de pescado del embalse de Urrá y a los tesistas-investigadores involucrados en los proyectos de investigación «Estimación de los parámetros biológicos básicos de peces comerciales del río Sinú-Fase I», Código FMV-01-01 y «Estimación de los parámetros biológicos básicos de peces comerciales del río Sinú-Fase III», Código FMV-01-06, de los cuales hace parte este trabajo. A la Universidad de Córdoba, por la financiación recibida.

\section{Literatura citada}

Agostinho AA, Ferretti CML, Gomes LC, Hahn NS, Suzuki HI, Fugi R, et al. 1997. Ictiofauna de dois reservatórios do rio Iguaçu em diferentes fases de colonização: Segredo e Foz do Areia. In: Agostinho AA, Gomes LC (eds.). Reservatório de Segredo: bases ecológicas para o manejo. Maringá: EDUEM. p. 257-92.

Agostinho AA, Miranda LE, Bini LM, Gomes LC, Thomaz SM, Suzuki HI. 1999. Patterns of colonization in neotropical reservoirs, and prognoses on aging. In: Tundisi JG,
Straskraba M (eds.). Theoretical reservoir ecology and its applications. Leiden: Backhuys Publishers. p. 22765.

Bagenal TB, Tesch FW. 1978. Age and growth. In: Bagenal TB (ed.). Methods for assessment of fish production in fresh waters. IBP Handbook $N^{\circ} 3$. Oxford: Blackwell Scientific Publications. p. 101-36.

Benedito-Cecilio E, Agostinho AA, Velho RCCM. 1997. Length-weight relationship of fishes caught in the Itaipu Reservoir, Paraná, Brazil. Naga ICLARM Quart. 20 (34): $57-61$.

Dahl G. 1971. Los peces del norte de Colombia. Bogotá: Inderena; $371 \mathrm{p}$.

De Hoyos IM. 2008. Relaciones talla-peso y factor de condición del Perico (Trachelyopterus badelif.c. Dahl, 1955) en el embalse de Urrá, Colombia. Trabajo de pregrado. Lorica: Programa de Acuicultura, Departamento de Ciencias Acuícolas, Facultad de Medicina Veterinaria y Zootecnia, Universidad de Córdoba $32 \mathrm{p}$.

FAO. 1998. Pesca continental. Orientaciones técnicas para la pesca responsable. Roma: Organización de las Naciones Unidas para la Agricultura y la Alimentación (FAO). 49 $\mathrm{p}$.

Gulland JA. 1983. Fish stock assessment: a manual of basic methods. Chichester: FAO/John Wiley and Sons. 223 p.

Hahn NS, Fugi R, Almeida VLL, Russo MR, Loureiro VE. 1997. Dieta e atividade alimentar de peixes do reservatório de Segredo. In: Agostinho AA, Gomes LC (eds.). Reservatório de Segredo: bases ecológicas para o manejo. Maringá: EDUEM. p. 141-62.

Lowe-McConnell RH. 1999. Estudos ecológicos de comunidades de peixes tropicais. São Paulo: Editora da Universidade de São Paulo. 535 p.

Luz-Agostinho Karla DG, Bini LM, Fugi R, Agostinho AA, Júlio Jr. HF. 2006. Food spectrum and trophic structure of the ichthyofauna of Corumbá reservoir, Paraná river Basin, Brazil. Neotrop Ichthyol 4 (1): 61-8.

Olaya-Nieto CW, Mercado T, Atencio-García VJ. 2000. Estimación del ictioplancton en el río Sinú, aguas arriba y aguas abajo de la presa. Informe final del Contrato Interadministrativo Urrá SA ESP-Universidad de Córdoba 011/2000. Montería: CINPIC-Departamento de Acuicultura. $91 \mathrm{p}$.

Olaya-Nieto CW, Brú-Cordero SB, Segura-Guevara F, Tordecilla-Petro G. 2004. Estimación de los parámetros biológicos básicos de peces comerciales del Río SinúFase I. Informe final. Lorica: Laboratorio de Investigación Biológico Pesquera-LIBP, Departamento de Acuicultura, Facultad de Medicina Veterinaria y Zootecnia, Universidad de Córdoba. 100 p.

Olaya-Nieto CW, Segura-Guevara FF, Tordecilla-Petro G, Appeldoorn RS. 2007. Estimación de los parámetros biológicos básicos de peces comerciales del río SinúFase II. Informe final. Lorica: Laboratorio de Investigación Biológico Pesquera-LIBP, Programa de Acuicultura, Departamento de Ciencias Acuícolas, Facultad de Medicina Veterinaria y Zootecnia, Universidad de Córdoba. 238 p. 
Pérez A, Olaya-Nieto CW, Segura-Guevara FF, Tordecilla-Petro G, Brú-Cordero SB. 2007. Relaciones longitud-peso de la Doncella, Ageneiosus pardalis (Pisces: Auchenipteridae), en la cuenca del río Sinú, Colombia. Dahlia 9: 53-61.

Ramírez Y, Saleme E. 2008. Biología reproductiva del Perico (Trachelyopterus badeli f.c. Dahl, 1955) en el río Sinú, Colombia. Trabajo de pregrado. Lorica: Programa de Acuicultura, Departamento de Ciencias Acuícolas, Facultad de Medicina Veterinaria y Zootecnia, Universidad de Córdoba. 33 p.

Ricker WE. Computation and interpretation of biological statistics of fish population. J Fish Res Board Can. 1975; 191: $1-382$.

Rodríguez A. 1998. Fish species composition before and after construction of a reservoir on the Guadalete River (SW Spain). Arch Hydrobiol. 142: 353-69.

Valderrama M, Garzón AC, Salas F, Villadiego P, Rangel B.
2002. Monitoreo ictiológico y pesquero del embalse de Urrá. Informe final año 2001 presentado a Urrá SA ESP. Montería: Fundación Bosques y Humedales. 107 p.

Valderrama M, Garzón AC, Salas F, Rangel B, Solano D, Fadul M. 2003. Monitoreo ictiológico y pesquero del embalse de Urrá. Informe final año 2002 presentado a Urrá SA ESP. Montería: Fundación Bosques y Humedales. 74 p.

Valderrama M, Salas F, Rangel B, Vertel C, Gómez D. 2004. Monitoreo ictiológico y pesquero del embalse de Urrá. Informe final año 2003 presentado a Urrá SA ESP. Montería: Fundación Bosques y Humedales. 94 p.

Valderrama M, Salas F, Solano D. 2006. Los peces y las pesquerías en el embalse de Urrá 2001-2005. Montería: Fundación Bosques y Humedales/Urrá SA ESP. 107 p.

Winemiller KO. 1986. Dynamic diversity in fish assemblages of tropical rivers. In: Cody ML, Smallwood R (eds.). Longterm studies of vertebrate communities. Orlando: Academic Press. p. 99-134. 